\title{
I finanziamenti europei per la ricerca
}

\author{
Ada Taratufolo ${ }^{1}$, Francesco Burrai \\ ${ }^{1}$ Professore a contratto, Università "La Sapienza" di Roma, Responsabile Ricerca e Formazione Continua SAIO, ASL Viterbo, Viterbo; Compo- \\ nente dell'Italian EDTNA/ERCA Research Center \\ ${ }^{2}$ Professore a contratto, Università di Bologna, Coordinatore Didattico, Azienda Ospedaliera Universitaria di Bologna, Bologna; Direttore Scien- \\ tifico dell'Italian EDTNA/ERCA Research Center
}

\begin{abstract}
The European funding for research
Among the eleven strategic objectives of the Common Strategic Framework established by the European Union, there are several key issues related to the development of research in Europe. The topics identified by the EC concern the strengthening of research, technological development and innovation and the improvement of access, use and quality of information and communications technology and of the investment in the skills and in continuous learning. The EC, to ensure the achievement of these objectives, arranges financing plans that can be accessed by the different countries, with specific procedures for the selection of the projects. It is fundamental, in a context of severe shortage of public funding for Italian research, to know what are the opportunities of the financing plans proposed by the EC and what are the ways to have access to these funds. The opportunities offered by the announcements of European funding are strategic and concrete ways for the development of a high level of nursing research in Italian nephrology and dialysis fields. A first exposure of the framework regarding the EC financing plans is the goal of this work.
\end{abstract}

Keywords: European funding, Nursing Research, Research, Research projects

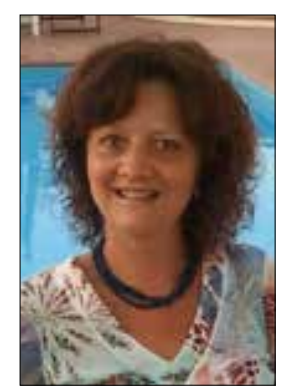

Ada Taratufolo

\section{Introduzione}

Nel Marzo del 2010, la Commissione Europea (CE) lancia la strategia EUROPA 2020 "per una crescita intelligente, sostenibile e inclusiva" (1). Con questo documento, la CE propone gli obiettivi e i criteri generali per la programmazione 2014-2020 (2), affrontando grandi sfide quali I'uscita dalla crisi, la globalizzazione delle relazioni economiche, il cambiamento climatico, la scarsità delle risorse (acqua, energia, materie prime), l'evoluzione demografica e i contrasti sociali.

Accepted: July 12, 2016

Published online: July 28, 2016

Indirizzo per la corrispondenza:

Dr.ssa Ada Taratufolo

Responsabile Area ricerca e formazione continua

c/o Cittadella della Salute

Via E. Fermi 15

01100 Viterbo

ada.taratufolo@asl.vt.it
EUROPA 2020 si incardina su tre priorità, concepite per rafforzarsi a vicenda:

1. crescita intelligente: sviluppare un'economia basata sulla conoscenza e sull'innovazione: Istruzione - Società digitale;

2. crescita sostenibile: promuovere un'economia più efficiente sotto il profilo delle risorse, più verde e più competitiva: Lotta al cambiamento climatico - Energia pulita ed efficiente - Competitività;

3. crescita inclusiva: promuovere un'economia con un alto tasso di occupazione che favorisca la coesione sociale e territoriale: Competenze - Lotta alla povertà.

Il perseguimento di queste priorità deve portare al raggiungimento di 5 obiettivi generali entro il 2020:

1. il $75 \%$ delle persone di età compresa tra i 20 e i 64 anni deve avere un lavoro;

2. il $3 \%$ del PIL dell'UE deve essere investito in Ricerca e Sviluppo (R\&S);

3. i traguardi " $20 / 20 / 20$ " in materia di clima/energia devono essere raggiunti (compreso un incremento del $30 \%$ della riduzione delle emissioni se le condizioni lo permettono);

4. il tasso di abbandono scolastico deve essere inferiore al $10 \%$ e almeno il $40 \%$ dei giovani deve essere laureato; 
5. 20 milioni di persone in meno devono essere a rischio di povertà. L'Unione Europea si avvale di diverse tipologie di strumenti finanziari, gestiti attraverso un sistema di "responsabilità condivisa" tra la Commissione Europea e le Autorità degli Stati Membri (gestione indiretta) o a livello centrale da parte della Commissione Europea (gestione diretta).

\section{Finanziamenti della Comunità Europea}

I fondi della Comunità Europea (UE) possono essere:

A. a gestione indiretta e comprendono i fondi strutturali e di investimento europei;

B. a gestione diretta e comprendono:

- i finanziamenti diretti UE noti anche come "programmi tematici" o "programmi comunitari";

- gli strumenti finanziari per l'assistenza esterna.

Questi fondi vengono, in genere, erogati tramite una procedura di selezione dei progetti presentati in seguito a una Call For Proposal (Bando di presentazione progetti che ha una scadenza prestabilita), pubblicata sulla Gazzetta Ufficiale dell'Unione Europea e sui siti web delle singole Direzioni Generali della Commissione Europea. II progetto deve essere presentato mediante un partenariato, con almeno tre partner provenienti da diversi Stati Membri.

I tre principali Fondi (di tipo indiretto) a cui si può accedere sono:

- il Fondo europeo di sviluppo regionale (FESR);

- il Fondo sociale europeo (FES);

- il Fondo di coesione (FS) (I'Italia non rientra tra i beneficiari di questo fondo).

Viene stabilito un Quadro Strategico Comune (QSC) per il FESR, il FSE e il FS, per promuovere un uso integrato dei fondi e realizzare obiettivi comuni (Politica di coesione 2014-2020: Investire nella crescita e nell'occupazione). II QSC è inteso dall'UE come uno strumento per assicurare una programmazione strategica coerente per tutti i Fondi, un quadro di riferimento unitario che assicuri il raggiungimento dei target di EUROPA 2020.

L'obiettivo dell'Unione Europea, attraverso i diversi strumenti finanziari è quello di promuovere la cooperazione e la sinergia sia all'interno che all'esterno dell'Europa e, soprattutto, di ridurre le disparità economiche, sociali e territoriali tra le varie Regioni europee. Le Regioni più povere dell'Europa ricevono la maggior parte dei finanziamenti, anche se tutte le Regioni europee possono usufruire di finanziamenti tramite i diversi meccanismi.

Il budget viene assegnato attraverso un sistema di "responsabilità condivisa" tra la Commissione Europea da una parte e le Autorità degli Stati Membri dall'altra.

Nei fondi indiretti, il rapporto con il beneficiario finale, pertanto, non è diretto, ma mediato da Autorità nazionali, regionali o locali che hanno il compito di programmare gli interventi, emanare i bandi e gestire le risorse comunitarie.

Nei fondi strutturali e di investimento europei i programmi sono, in pratica, gestiti dalle Autorità nazionali e locali, ma finanziati dalla Commissione Europea attraverso la seguente modalità:

la Commissione UE negozia e approva i programmi di sviluppo proposti dai singoli Stati Membri e stanzia le risorse finanziarie;

- gli Stati Membri e le Regioni gestiscono i programmi e li attuano attraverso la selezione, il controllo e la valutazione dei progetti;

- la Commissione viene coinvolta nella fase di monitoraggio, si impegna nel pagare le spese approvate ed effettua verifiche attraverso un sistema di controllo.

Per ogni Programma Operativo, lo Stato Membro nomina:

- un'Autorità di gestione (un'Autorità pubblica o un organismo pubblico-privato, nazionale, regionale o locale designato dallo Stato Membro per gestire il Programma Operativo); un'Autorità di certificazione (un'Autorità pubblica o un organismo pubblico-privato, nazionale, regionale o locale designato dallo Stato Membro per certificare le dichiarazioni di spesa e le domande di pagamento prima del loro invio alla Commissione);

- un'Autorità di audit (un'Autorità pubblica o un organismo pubblico-privato, nazionale, regionale o locale funzionalmente indipendente dall'Autorità di Gestione e dall'Autorità di Certificazione, designato dallo Stato Membro per ciascun programma e responsabile della verifica dell'efficace funzionamento del sistema di gestione e controllo).

I Fondi diretti, invece, sono gestiti direttamente dalle diverse Direzioni Generali della Commissione Europea o da Agenzie da essa delegate. Si tratta di sovvenzioni o versamenti di natura non commerciale, che devono essere integrati da risorse proprie dei beneficiari. Questo tipo di finanziamenti richiede la costituzione di partenariati transnazionali. Come già anticipato, i Fondi diretti comprendono sia i programmi tematici sia gli strumenti di assistenza esterna; la Commissione Europea trasferisce gli importi direttamente ai beneficiari del progetto. Sono conosciuti anche come "programmi comunitari", come Horizon 2020 ed Erasmust Europa Creativa, con l'obiettivo di finanziare anche la ricerca.

\section{Come poter accedere ai fondi europei}

Prima cosa da fare è leggere il regolamento pubblicato sulla Gazzetta Ufficiale dell'Unione Europea, per poter valutare la possibilità di partecipare a un programma comunitario (3). La "Call For Proposer" contiene informazioni fondamentali amministrative, ma anche finanziarie, sul progetto che la comunità intende finanziare, i criteri di ammissibilità del progetto e di selezione, come anche il termine per la presentazione delle proposte.

La "Call For Proposer" viene sempre accompagnata dalla 
"Guide for Applicants", che fornisce indicazioni dettagliate su:

- modalità di presentazione delle domande;

- procedura di selezione delle proposte;

- condizioni finanziarie della sovvenzione;

- criteri di valutazione delle proposte progettuali;

- categorie di spesa ammissibili;

- modalità di pagamento e natura del contratto.

Ogni proposta presentata deve soddisfare cinque principi di base (4), per poter essere finanziata:

1) transnazionalità: i progetti devono dimostrare un chiaro interesse comunitario e coinvolgere organismi di diversi Stati UE e la composizione minima della partnership varia a seconda dei programmi ed è indicata nella guida al proponente.

2) cofinanziamento: la Commissione Europea non finanzia mai la totalità delle spese sostenute dai beneficiari della sovvenzione per l'esecuzione del progetto; gli organismi beneficiari, infatti, sono tenuti a finanziare in parte il proprio progetto (in quote che variano dal $50 \%$ all' $80 \%$ ), utilizzando risorse proprie e quelle dei partner;

3) non retroattività: la sovvenzione non può essere concessa per finanziare attività svolte, anche se parzialmente, prima della firma del contratto;

4) assenza di profitto: le attività oggetto della sovvenzione non possono generare profitto per le organizzazioni beneficiarie. Questo significa che il bilancio tra entrate e spese previste dal progetto deve sempre risultare in pareggio;

5) non cumulabilità: il progetto o una parte di esso non possono essere sostenuti da due o più sovvenzioni comunitarie.

Importante è, inoltre, individuare la Direzione Generale (DG) competente e verificare la congruenza degli obiettivi del progetto con quelli del programma comunitario di riferimento; inoltre, bisogna valutare la pertinenza del progetto in relazione a temi o a problematiche che la Commissione Europea reputa prioritari e, soprattutto, la composizione della partnership, perché questi possono variare sensibilmente a seconda dei programmi. Un progetto viene finanziato dalla Commissione Europea anche in ragione del fatto che la sua implementazione genera impatti positivi nella sua dimensione transnazionale.

\section{Modalità di finanziamento}

La Commissione Europea versa l'importo della sovvenzione attraverso formule diverse, che dipendono dal programma comunitario e, soprattutto, dalla durata del progetto.

In linea generale, la formula più utilizzata è quello del pagamento in tre rate:

1. un anticipo (pre-payment) corrispondente al 30\%-40\% dell'intero contributo richiesto, versato sul conto del beneficiario 30 giorni dopo la firma del contratto di sovvenzione;
2. un pagamento intermedio (mid term payment), pari a circa il $25 \%-30 \%$ del contributo totale, che viene versato a seguito dell'approvazione da parte della Commissione Europea del report intermedio del progetto, documento che descrive lo stato di avanzamento delle attività, con un rendiconto finanziario di quanto è stato speso da ogni partecipante al progetto;

3. un pagamento finale (final payment), a seguito dell'approvazione finale da parte della Commissione Europea del report finale e della dichiarazione conclusiva dei costi sostenuti.

\section{Conclusioni}

Negli ultimi anni, il finanziamento pubblico della ricerca ha subito drastici tagli e l'Italia si posiziona negli ultimi posti tra i paesi europei nella percentuale del prodotto interno lordo investito in ricerca. Questa situazione ha prodotto diverse condizioni: 1) la "fuga" dei migliori ricercatori italiani verso Università e Centri di Ricerca esteri; 2) incremento del numero dei progetti di ricerca italiani nei bandi europei e internazionali; 3 ) incremento del finanziamento della ricerca da parte di aziende private, associazioni scientifiche e associazioni dei pazienti; 4) incremento dei GRANT messi a bando dai Collegi IPASVI italiani. Davanti a questo panorama, appare strategico, per la ricerca infermieristica italiana in nefrologia, aprirsi verso una fondamentale e necessaria mentalità scientifica, caratterizzata da un rigore metodologico, attraverso l'acquisizione di competenze nella ricerca quantitativa, qualitativa e mixed method. Solo queste competenze metodologiche possono incrementare la qualità della ricerca infermieristica italiana in nefrologia e per poter, conseguentemente, essere competitivi scientificamente, con maggiori possibilità di accedere ai finanziamenti per la ricerca sia italiani che europei.

\section{Disclosures}

Financial support: No financial support was received for this submission. Conflict of interest: The authors have no conflict of interest.

\section{Bibliografia}

1. Comunicazione della Commissione COM (2010) 2020 del 3 Marzo 2010. La strategia globale e i relativi obiettivi sono stati discussi dal Parlamento Europeo e approvati dal Consiglio Europeo in occasione dei vertici di Marzo e Giugno 2010. Ulteriori informazioni sono disponibili all'indirizzo web: http://ec.europa. eu/europe2020/index_it.

2. Politica di coesione 2014-2020: Investire nella crescita e nell'occupazione. http://europalavoro.lavoro.gov.it/.../politicacoesione-2014-2020-pubb-regolamenti.pdf.

3. http://www.ilsole24ore.com/art/economia/2014-04-17/ledieci-mosse-accedere-finanziamenti-diretti-europei-090420. shtml?uuid=ABE4ckBB\& $p=3$.

4. Guida alle opportunità di finanziamento dell'UE 2014-2020 Formez (bozza Luglio 2013). http://www.slideshare.net/formezeu/guida-alle-opportunit-difinanziamento-dellunione-europea-2014-2020. http://europa.formez.it/content/guida-alle-opportunita-finanziamento-dellunione-europea-2014-2020. 